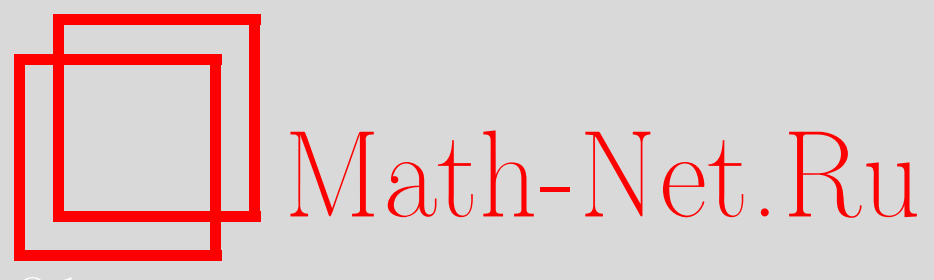

С. В. Манаков, П. М. Сантини, Иерархия интегрируемых уравнений в частных производных в размерности $2+1$, ассоциированная с однопараметрическими семействами одномерных векторных полей, ТМФ, 2007, том 152, номер 1, 147-156

DOI: https://doi.org/10.4213/tmf6076

Использование Общероссийского математического портала Math-Net.Ru подразумевает, что вы прочитали и согласны с пользовательским соглашением http://www . mathnet.ru/rus/agreement

Параметры загрузки:

IP : 3.82 .47 .9

26 апреля 2023 г., 17:09:05

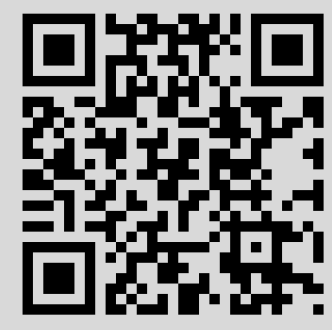




\title{
ИЕРАРХИЯ ИНТЕГРИРУЕМЫХ УРАВНЕНИЙ В ЧАСТНЫХ ПРОИЗВОДНЫХ В РАЗМЕРНОСТИ $2+1$, АССОЦИИРОВАННАЯ С ОДНОПАРАМЕТРИЧЕСКИМИ СЕМЕЙСТВАМИ ОДНОМЕРНЫХ ВЕКТОРНЫХ ПОЛЕЙ
}

\begin{abstract}
Введена иерархия интегрируемых уравнений в частных производных в размерности $2+1$, возникающая в результате коммутации однопараметрического семейства векторных полей. С помощью метода обратной задачи рассеяния построено формальное решение соответствующих задач Коши для однопараметрических семейств векторных полей. Благодаря тому что пространство собственных функций является кольцом, обратная задача может быть сформулирована тремя различными способами; в частности, одна из формулировок соответствует линейному интегральному уравнению для функций Йоста, а другая представляет собой скалярную нелинейную задачу Римана для подходящих аналитических собственных функций.
\end{abstract}

Ключевые слова: интегрируемые системы, метод обратной задачи рассеяния, обратное спектральное преобразование, семейства векторных полей, нелинейная задача Римана.

\section{1. ВВЕДЕНИЕ}

В настоящей работе вводится иерархия интегрируемых дифференциальных уравнений в частных производных (ДУЧП) в размерности $2+1$, возникающая в результате коммутации однопараметрических семейств одномерных векторных полей, и строится формальное решение соответствующих задач Коши с помощью недавно предложенного в работах [1], [2] метода обратной задачи рассеяния (ОЗР) для векторных полей.

Первым нетривиальным членом такой иерархии является уравнение

$$
v_{x t}+v_{y y}=v_{y} v_{x x}-v_{x} v_{x y}, \quad v=v(x, y, t) \in \mathbb{R}, \quad x, y, t \in \mathbb{R},
$$

* Институт теоретической физики им. Л. Д. Ландау РАН, Москва, Россия.

E-mail: manakov@itp.ac.ru

†Dipartimento di Fisica, Università di Roma "La Sapienza" and Istituto Nazionale di Fisica Nucleare, Sezione di Roma 1 Piazz.le Aldo Moro 2, I-00185 Roma, Italy.

E-mail: paolo.santini@roma1.infn.it 
введенное в работе [3] и изученное в работах [3]-[5] с различных точек зрения, которое допускает операторное представление

$$
[\hat{L}, \widehat{M}]=0
$$

в терминах двумерных дифференциальных операторов

$$
\begin{aligned}
\hat{L} & \equiv \partial_{y}+\left(p+v_{x}\right) \partial_{x} \\
\widehat{M} & \equiv \partial_{t}+\left(p^{2}+p v_{x}-v_{y}\right) \partial_{x}
\end{aligned}
$$

где $p$ - постоянный параметр.

Уравнение (1) представляет собой редукцию $u=0$ недавно введенной [1] интегрируемой системы

$$
\begin{aligned}
u_{x t}+u_{y y} & =-\left(u u_{x}\right)_{x}-v_{x} u_{x y}+v_{y} u_{x x}, \\
v_{x t}+v_{y y} & =-u v_{x x}-v_{x} v_{x y}+v_{y} v_{x x},
\end{aligned} \quad u, v \in \mathbb{R}, \quad x, y, t \in \mathbb{R},
$$

которая допускает операторное представление $[\tilde{L}, \widetilde{M}]=0$ в терминах следующих двумерных векторных полей:

$$
\begin{aligned}
\tilde{L} & \equiv \partial_{y}+\left(p+v_{x}\right) \partial_{x}-u_{x} \partial_{p} \\
\widetilde{M} & \equiv \partial_{t}+\left(p^{2}+p v_{x}+u-v_{y}\right) \partial_{x}+\left(-p u_{x}+u_{y}\right) \partial_{p}
\end{aligned}
$$

Отметим, что редукция $v=0$ системы (4) представляет собой знаменитое бездисперсионное уравнение Кадомцева-Петвиашвили (КП) (или уравнение ХохловаЗаболотской)

$$
u_{t x}+u_{y y}+\left(u u_{x}\right)_{x}=0, \quad u=u(x, y, t) \in \mathbb{R}, \quad x, y, t \in \mathbb{R},
$$

которое возникает в различных задачах математической физики и интенсивно исследуется в современной литературе (см., например, работы [6]-[11]).

ОЗР для однопараметрического семейства многомерных векторных полей недавно была предложена и использована для построения формального решения задачи Коши широкого класса нелинейных ДУЧП в произвольных размерностях [1], включая уравнение Плебански [12] и систему (4) [2]. Гамильтоново ограничение на ассоциированные спектральные данные, приводящее к редукции системы (4) к уравнению Плебански и к бездисперсионному уравнению КП, также было найдено в работах [1], [2]. Отметим, что в случае гамильтоновых векторных полей элегантная альтернативная схема интегрирования уже известна из литературы [7].

Заметим также, что, поскольку рассматриваемая в настоящей работе иерархия ДУЧП возникает в результате коммутации однопараметрических семейств векторных полей, то в соответствии с развитой в работах [1], [2] теорией ассоциированная обратная задача может быть сформулирована в виде скалярной нелинейной задачи Римана. 


\section{2. ИЕРАРХИЯ ДУЧП, АССОЦИИРОВАННАЯ С ОПЕРАТОРОМ $\hat{L}$}

Уравнение (1) является первым нетривиальным членом (соответствующим $n=2$ ) следующей иерархии ДУЧП в размерности $2+1$, возникающей в результате коммутации одномерных векторных полей:

$$
v_{t_{n}}+\widehat{\mathcal{Q}}^{n} v_{x}=0, \quad n \in \mathbb{N},
$$

где $\widehat{\mathcal{Q}}$ - оператор рекурсии

$$
\widehat{\mathcal{Q}} \equiv \partial_{x}^{-1}\left(v_{x x}-\partial_{y}-v_{x} \partial_{x}\right)=v_{x}-\partial_{x}^{-1} \partial_{y}-2 \partial_{x}^{-1} v_{x} \partial_{x} .
$$

Первые несколько потоков суть

$$
\begin{gathered}
v_{t_{0}}+v_{x}=0, \\
v_{t_{1}}-v_{y}=0, \\
v_{x t_{2}}+v_{y y}-v_{y} v_{x x}+v_{x} v_{x y}=0, \\
v_{x x t_{3}}-\left(v_{y}+v_{x}^{2}\right)_{y y}+\left[v_{x y}\left(v_{y}-v_{x}^{2}\right)+v_{x x} \partial_{x}^{-1}\left(v_{y}+v_{x}^{2}\right)_{y}\right]_{x}=0 .
\end{gathered}
$$

Эта иерархия допускает операторное представление

$$
\left[\hat{L}, \widehat{M}_{n}\right]=0, \quad n \in \mathbb{N},
$$

где оператор $\hat{L}$ определен первым из уравнений (3), а однопараметрическое семейство векторных полей $\widehat{M}_{n}$ определяется формулой

$$
\widehat{M}_{n} \equiv \partial_{t_{n}}+\left(p^{n}+\sum_{k=0}^{n-1} p^{k} A_{n-k}^{(n)}\right) \partial_{x}
$$

в которой

$$
A_{k+1}^{(n)}=\widehat{\mathcal{Q}} A_{k}^{(n)}, \quad 1 \leqslant k \leqslant n-1, \quad A_{1}^{(n)}=v_{x} .
$$

Чтобы показать это, подставим выражение для $\hat{L}$ из $(3)$ и формулу $(11)$ в представление (10) и получим следующее полиномиальное (по $p$ ) уравнение:

$$
v_{x t}+v_{x x}\left(p^{n}+\sum_{k=0}^{n-1} p^{k} A_{n-k}^{(n)}\right)=\sum_{k=0}^{n-1} p^{k}\left(A_{n-k}^{(n)}\right)_{y}+\left(p+v_{x}\right) \sum_{k=0}^{n-1} p^{k}\left(A_{n-k}^{(n)}\right)_{x},
$$

которое должно выполняться для любого $p$. Приравнивая к нулю коэффициенты при всех степенях $p$, получаем уравнения (12) и (7).

Поскольку оператор $\widehat{\mathcal{Q}}$ не зависит явно от $x$, его производная Ли по направлению $v_{x}$, первое векторное поле иерархии, равна нулю; кроме того, можно проверить, что оператор $\widehat{\mathcal{Q}}$ является оператором Нийенхейса (наследственным оператором). Поэтому все потоки (7) коммутируют друг с другом [13], [14].

Заметим, что алгебраическая теория для двумерного оператора рекурсии $\widehat{\mathcal{Q}}$ концептуально более похожа на теорию одномерных операторов рекурсии, ассоциированных с системами типа уравнения Кортевега-де Фриза и нелинейного уравнения 
Шредингера [13], чем на теорию двумерных операторов рекурсии, ассоциированных с системами типа уравнений КП и Дэви-Стюартсона [15]. Тем не менее на данный момент результаты относительно гамильтонова и бигамильтонова [14] характера иерархии (7) не известны. Неявное представление иерархии, не использующее оператор рекурсии, дано в работе [5].

\section{3. МЕТОД ОЗР}

Рассмотрим теперь задачу Коши для иерархии (7) и, в частности, для уравнения (1) в классе быстроубывающих вещественных потенциалов $v$ :

$$
v\left(x, y, t_{n}\right) \rightarrow 0, \quad\left(x^{2}+y^{2}\right) \rightarrow \infty, \quad v \in \mathbb{R}, \quad(x, y) \in \mathbb{R}^{2}, \quad t_{n}>0,
$$

интерпретируя $t_{n}$ как время, а другие две переменные $x, y$ - как пространственные переменные. Чтобы решить такую задачу Коши методом ОЗР, развитым в работах [1], [2], построим обратное спектральное преобразование для оператора $\hat{L}$ в классе быстроубывающих вещественных потенциалов, интерпретируя операторы $\widehat{M}_{n}$ как временнь́е операторы.

Из операторного представления (10) вытекает существование общих собственных функций $f(x, y, t, p)$ операторов $\hat{L}, \widehat{M}_{n}$ (пары Лакса):

$$
\hat{L} f=0, \quad \widehat{M}_{n} f=0, \quad n \in \mathbb{N} .
$$

Поскольку пара Лакса (15) образована векторными полями, то пространство собственных функций есть кольщо: если $f_{1}, f_{2}$ - два решения для пары Лакса (15), то произвольная дифференцируемая функция от этих решений снова является решением (15).

3.1. Прямая задача. Из условия (14) для потенциала $v$ следует, что если $f-$ решение уравнения $\hat{L} f=0$, то

$$
f(x, y, p) \rightarrow f_{ \pm}(\xi, p), \quad y \rightarrow \pm \infty, \quad \xi:=x-p y
$$

т.е. асимптотически $f$ является произвольной функцией переменных $\xi=x-p y$ и $p$.

Главную роль в теории играет вещественная собственная функция Йоста $\varphi(x, y, p)$ - решение уравнения $\hat{L} \varphi=0$, однозначно определяемое асимптотикой

$$
\varphi(x, y, p) \rightarrow \xi, \quad y \rightarrow-\infty
$$

или, эквивалентно, характеризующаяся линейным интегральным уравнением $\varphi=$ $\xi+\widehat{G}\left(-v_{x} \varphi_{x}\right)$ для функции Грина $G(x, y, p)=\theta(y) \delta(x-p y)$.

Предел $y=+\infty$ функции Йоста $\varphi$ определяет обычные данные рассеяния $\sigma$ для оператора $\hat{L}$ :

$$
\lim _{y \rightarrow+\infty} \varphi(x, y, p) \equiv \mathcal{S}(\xi, p)=\xi+\sigma(\xi, p) .
$$

Прямая задача есть переход от вещественного потенциала $v$ - функции двух вещественных переменных $x, y-$ к вещественным данным рассеяния $\sigma$ - функции двух 
вещественных переменных $\xi, p$. В пределе малого поля это отображение сводится к преобразованию Радона [16]:

$$
\sigma(\xi, p)=-\int_{\mathbb{R}} v_{\xi}(\xi+p y, y) d y .
$$

Важную роль в теории обратного спектрального преобразования для векторного поля $\hat{L}$ играют также аналитические собственные функции $\psi_{ \pm}(x, y, p)-$ решения уравнения $\hat{L} \psi_{ \pm}=0$, удовлетворяющие интегральным уравнениям

$$
\psi_{ \pm}(x, y, p)=-\int_{\mathbb{R}^{2}} d x^{\prime} d y^{\prime} G_{ \pm}\left(x-x^{\prime}, y-y^{\prime}, p\right) v_{x^{\prime}}\left(x^{\prime}, y^{\prime}\right) \psi_{ \pm_{x^{\prime}}}\left(x^{\prime}, y^{\prime}, p\right)+\xi
$$

где $G_{ \pm}-$аналитические функции Грина

$$
G_{ \pm}(x, y, p)= \pm \frac{1}{2 \pi i[x-(p \pm i \epsilon) y]} .
$$

Из аналитических свойств функций Грина $G_{ \pm}(x, y, p)$ в комплексной плоскости $p$ следует, что функции $\psi_{+}(x, y, p)$ и $\psi_{-}(x, y, p)$ являются аналитическими соответственно в верхней и нижней полуплоскостях комплексной плоскости $p$ и при больших значениях $p$ имеют асимптотики

$$
\psi_{ \pm}(x, y, p)=\xi-\frac{v(x, y)}{p}+O\left(\frac{1}{p^{2}}\right), \quad|p| \gg 1 .
$$

Важно отметить, что аналитические функции Грина (21) обладают следующими асимптотиками при $y \rightarrow \pm \infty$ :

$$
\begin{array}{ll}
G_{ \pm}\left(x-x^{\prime}, y-y^{\prime}, p\right) \rightarrow \pm \frac{1}{2 \pi i\left[\xi-\xi^{\prime} \mp i \epsilon\right]}, & y \rightarrow+\infty, \\
G_{ \pm}\left(x-x^{\prime}, y-y^{\prime}, p\right) \rightarrow \pm \frac{1}{2 \pi i\left[\xi-\xi^{\prime} \pm i \epsilon\right]}, & y \rightarrow-\infty,
\end{array}
$$

из чего вытекает, что $(y=+\infty)$-асимптотики функиий $\psi_{+} u \psi_{-}$являются аналитическими соответственно в нижней и верхней полуплоскостях комплексной плоскости $\xi$, тогда как $(y=-\infty)$-асимптотики этих функиий, наоборот, являются аналитическими соответственно в верхней и нижней полуплоскостях комплексной плоскости $\xi$ (похожие свойства описывались впервые в работе [17] и позднее в работах [1], [2]).

Собственная функция Йоста $\varphi$ и постоянная собственная функция $p$ образуют базис в кольце решений уравнения $\hat{L} f=0$; таким образом, любое решение $f$ уравнения $\hat{L} f=0$ является функцией от $\varphi$ и $p$. В частности, аналитические собственные функции $\psi_{ \pm}$обладают представлениями

$$
\psi_{ \pm}=\mathcal{K}_{ \pm}(\varphi, p)=\varphi+\chi_{ \pm}(\varphi, p)
$$

определяющими спектральные данные $\chi_{ \pm}$. Поскольку предел выражения (24) при $y \rightarrow-\infty$ имеет вид

$$
\lim _{y \rightarrow-\infty} \psi_{ \pm}-\xi=\chi_{ \pm}(\xi, p)
$$


то из указанных выше аналитических свойств левой части равенства (25) в комплексной плоскости $\xi$ следует, что функции $\chi_{+}(\xi)$ и $\chi_{-}(\xi)$, убывая при $\xi \sim \infty$ как $O\left(\xi^{-1}\right)$, являются аналитическими соответственно в верхней и нижней полуплоскостях комплексной плоскости $\xi$. Поэтому фурье-преобразования этих функций $\tilde{\chi}_{+}(\omega)$ и $\tilde{\chi}_{-}(\omega)$ имеют носители соответственно на положительной и отрицательной полуосях $\omega$.

Спектральные данные $\chi_{ \pm}$могут быть построены из данных рассеяния $\sigma$ при помощи линейных интегральных уравнений

$$
\begin{aligned}
\tilde{\chi}_{+}(\omega, p)+\theta(\omega)\left(\tilde{\sigma}(\omega, p)+\int_{\mathbb{R}} d \eta \tilde{\chi}_{+}(\eta, p) Q(\eta, \omega, p)\right) & =0, \\
\tilde{\chi}_{-}(\omega, p)+\theta(-\omega)\left(\tilde{\sigma}(\omega, p)+\int_{\mathbb{R}} d \eta \tilde{\chi}_{-}(\eta, p) Q(\eta, \omega, p)\right) & =0,
\end{aligned}
$$

которые включают фурье-преобразования $\tilde{\sigma}$ и $\tilde{\chi}_{ \pm}$функций $\sigma$ и $\chi_{ \pm}$:

$$
\tilde{\sigma}(\omega, p)=\int_{\mathbb{R}} d \xi \sigma(\xi, p) e^{-i \omega \xi}, \quad \tilde{\chi}_{ \pm}(\omega, p)=\int_{\mathbb{R}} d \xi \chi_{ \pm}(\xi, p) e^{-i \omega \xi},
$$

и ядро

$$
Q(\eta, \omega, p)=\int_{\mathbb{R}} \frac{d \xi}{2 \pi} e^{i(\eta-\omega) \xi}\left[e^{i \eta \sigma(\xi, p)}-1\right] .
$$

Чтобы доказать это, вычислим сначала (24) при $y=+\infty$; имеем

$$
\lim _{y \rightarrow \infty} \psi_{ \pm}-\xi=\sigma(\xi, p)+\chi_{ \pm}(\xi+\sigma(\xi, p), p) .
$$

Применяя интегральный оператор $\int_{\mathbb{R}} d \xi e^{-i \omega \xi}$ с $\omega>0$ и $\omega<0$ к уравнениям $(29)$ с индексами + и - , соответственно, используя вышеуказанные аналитические свойства и фурье-представления для функций $\chi_{ \pm}$и $\sigma$, получаем уравнения $(26)$.

Из вещественности потенциала $v \in \mathbb{R}$ вытекает, что $\bar{\varphi}=\varphi, \bar{\psi}_{+}=\psi_{-}$для $p \in \mathbb{R}$; следовательно, $\bar{\sigma}=\sigma, \bar{\chi}_{+}=\chi_{-}$.

3.2. Обратная задача. То обстоятельство, что пространство собственных функций является кольцом, позволяет, как и в работах [1], [2], построить три различных варианта обратной задачи; в основе всех трех вариантов лежат уравнения (24).

Первый вариант получается следующим образом. Вычитая $\xi$ из уравнений (24) с индексами + и - , применяя к полученным уравнениям аналитические проекторы $\widehat{P}_{+}$и $\widehat{P}_{-}$,

$$
\widehat{P}_{ \pm} \equiv \pm \frac{1}{2 \pi i} \int_{\mathbb{R}} \frac{d p^{\prime}}{p^{\prime}-(p \pm i \epsilon)},
$$

соответственно, и складывая полученные в результате уравнения, приходим к следующему нелинейному интегральному уравнению для собственной функции Йоста $\varphi$ :

$$
\begin{aligned}
\varphi(x, y, p) & +\frac{1}{2 \pi i} \int_{\mathbb{R}} \frac{d p^{\prime}}{p^{\prime}-(p+i \epsilon)} \chi_{-}\left(\varphi\left(x, y, p^{\prime}\right), p^{\prime}\right)- \\
& -\frac{1}{2 \pi i} \int_{\mathbb{R}} \frac{d p^{\prime}}{p^{\prime}-(p-i \epsilon)} \chi_{+}\left(\varphi\left(x, y, p^{\prime}\right), p^{\prime}\right)=x-p y .
\end{aligned}
$$


Как только функция $\varphi$ восстановлена из уравнения (31) по заданным спектральным данным $\chi_{ \pm}(\xi, p)$, аналитические собственные функции восстанавливаются по формуле (24), а потенциал $v$ - из уравнения (22). Такая процедура обращения впервые была предложена в работе [18] и использовалась также в работах [1], [2].

Второй вариант представляет собой линейный аналог нелинейной задачи (31), который получается в результате экспоненцирования функиии Йоста и аналитических собственных функций, использованных выше. Рассмотрим функции

$$
\Phi(x, y, p ; \alpha) \equiv e^{i \alpha \varphi(x, y, p)}, \quad \Psi_{ \pm}(x, y, p ; \alpha) \equiv e^{i \alpha \psi_{ \pm}(x, y, p)}, \quad \alpha \in \mathbb{R} .
$$

Благодаря тому что пространство собственных функций является кольцом, функции $\Phi(x, y, p ; \alpha)$ и $\Psi_{ \pm}(x, y, p ; \alpha)$ также являются собственными функциями: функция $\Phi(x, y, p ; \alpha)$ есть собственная функция Йоста, определяемая асимптотикой $\Phi \rightarrow e^{i \alpha \xi}$, $y \rightarrow-\infty$, а функции $\Psi_{ \pm}(x, y, p ; \alpha)$ суть собственные функции, аналитические соответственно в верхней и нижней полуплоскостях комплексной плоскости $p$ с асимптотиками $\Psi_{ \pm}=e^{i \alpha \xi}\left[1-(\alpha v) / p+O\left(p^{-2}\right)\right]$.

Экспоненцируя представления (24), получаем линейные разложения аналитических собственных функций $\Psi_{ \pm}$в терминах собственной функции Йоста $\Phi$ :

$$
\Psi_{ \pm}(x, y, p ; \alpha)=\Phi(x, y, p ; \alpha)+\int_{\mathbb{R}} d \beta K_{ \pm}(\alpha, \beta, p) \Phi(x, y, p ; \beta),
$$

где новые спектральные данные $K_{ \pm}$определяются через спектральные данные $\chi_{ \pm}$ формулой

$$
K_{ \pm}(\alpha, \beta, p) \equiv \int_{\mathbb{R}} \frac{d \xi}{2 \pi} e^{i(\alpha-\beta) \xi}\left[e^{i \alpha \chi_{ \pm}(\xi, p)}-1\right] .
$$

Умножая уравнения (33) с индексами + и - на $e^{-i \alpha \xi}$, вычитая 1 , применяя соответственно проекторы $\widehat{P}_{-}$и $\widehat{P}_{+}$и складывая полученные в результате уравнения, получаем следующее линейное интегралъное уравнение для функции $\Phi$ :

$$
\begin{aligned}
\Phi(x, y, p ; \alpha) & +\frac{1}{2 \pi i} \int_{\mathbb{R}} \frac{d p^{\prime}}{p^{\prime}-(p+i \epsilon)} \int_{\mathbb{R}} d \beta K_{-}\left(\alpha, \beta, p^{\prime}\right) \Phi\left(x, y, p^{\prime} ; \beta\right) e^{i \alpha\left(p^{\prime}-p\right) y}- \\
& -\frac{1}{2 \pi i} \int_{\mathbb{R}} \frac{d p^{\prime}}{p^{\prime}-(p-i \epsilon)} \int_{\mathbb{R}} d \beta K_{+}\left(\alpha, \beta, p^{\prime}\right) \Phi\left(x, y, p^{\prime} ; \beta\right) e^{i \alpha\left(p^{\prime}-p\right) y}=e^{i \alpha(x-p y)} .
\end{aligned}
$$

Как только функция $\Phi$ восстановлена из уравнения (35), а также из уравнения (33) получены функции $\Psi_{ \pm}$, потенциалы восстанавливаются обычным образом по асимптотикам функций $\Psi_{ \pm}$.

Из условия действительности $(v \in \mathbb{R})$ следует, что $\overline{\Phi(\alpha)}=\Phi(-\alpha), \overline{\Psi_{+}(\alpha)}=$ $\Psi_{-}(-\alpha)$ при $\alpha, p \in \mathbb{R}$. Соответственно $\overline{K_{+}(\alpha, \beta)}=K_{-}(-\alpha,-\beta)$.

Третий вариант обратной задачи представляет собой задачу Римана. Решая алгебраическую систему (24) с индексом - относительно $\varphi: \varphi=\mathcal{L}\left(\psi_{-}, p\right)$ (предполагая локальную обратимость) и подставляя это выражение в алгебраическую систему (24) с индексом + , получаем представление аналитической собственной функции $\psi_{+}$через аналитическую собственную функцию $\psi_{-}$:

$$
\psi_{+}=\mathcal{R}\left(\psi_{-}, p\right)=\psi_{-}+R\left(\psi_{-}, p\right), \quad p \in \mathbb{R},
$$


где $\mathcal{R}\left(\psi_{-}, p\right)=\mathcal{K}_{+}\left(\mathcal{L}\left(\psi_{-}, p\right), p\right)$, которое определяет скалярную нелинейную задачу Римана на вещественной оси $p$. Данные задачи Римана $\mathcal{R}$ строятся, таким образом, из данных $\mathcal{K}_{ \pm}$посредством алгебраических действий. Наоборот, по заданным данным $R$ строятся решения $\psi_{ \pm}$нелинейной задачи Римана $(36)$, и по асимптотикам (22) восстанавливается потенциал $v$.

Условие действительности для данных задачи Римана $\mathcal{R}$ принимает следующий вид: $\mathcal{R}(\overline{\mathcal{R}(\bar{\xi}, p)}, p)=\xi$ для любого $\xi$ и $p \in \mathbb{R}$.

ЗАмечАниЕ. Одевающие схемы могут быть прямо выведены из трех различных обратных задач, представленных в этой работе.

3.3. Эволюция спектральных данных во времени. Если потенциал $v$ эволюционирует во времени согласно уравнению (1), то зависимость спектральных данных $\sigma, \chi_{ \pm}, R$ и $K_{ \pm}$от времени описывается следующими явными формулами:

$$
\begin{array}{cl}
\sigma(\xi, p, t)=\sigma\left(\xi-p^{2} t, p, 0\right), & \mathcal{S}(\xi, p, t)=\xi+\sigma\left(\xi-p^{2} t, p, 0\right), \\
\chi_{ \pm}(\xi, p, t)=\chi_{ \pm}\left(\xi-p^{2} t, p, 0\right), & \mathcal{K}_{ \pm}(\xi, p, t)=\xi+\chi_{ \pm}\left(\xi-p^{2} t, p, 0\right), \\
R(\xi, p, t)=R\left(\xi-p^{2} t, p, 0\right), & \mathcal{R}(\xi, p, t)=\xi+R\left(\xi-p^{2} t, p, 0\right), \\
K_{ \pm}(\alpha, \beta, p, t)=K_{ \pm}(\alpha, \beta, p, 0) e^{i(\alpha-\beta) p^{2} t} .
\end{array}
$$

Чтобы доказать это, заметим прежде всего, что функция

$$
\phi(x, y, t, p) \equiv \varphi(x, y, t, p)-p^{2} t
$$

является общей собственной функцией Йоста операторов $\hat{L}$ и $\widehat{M}$. Предел $y=+\infty$ уравнения $\widehat{M} \phi=0$ дает уравнение $\sigma_{t}+p^{2} \sigma_{\xi}=0$, решением которого является первая пара равенств из (37). Аналогично функции

$$
\pi_{ \pm}(x, y, t, p) \equiv \psi_{ \pm}(x, y, t, p)-p^{2} t
$$

являются общими аналитическими собственными функциями операторов $\hat{L}$ и $\widehat{M}$, поэтому

$$
\pi_{ \pm}=\check{\mathcal{K}}_{ \pm}(\phi, p), \quad \pi_{+}=\check{\mathcal{R}}\left(\pi_{-}, p\right)
$$

для некоторых функций $\check{\mathcal{K}}_{ \pm}$и $\check{\mathcal{R}}$, зависящих от переменных $x, y, t$ только через функции $\phi$ и $\pi_{-}$. Сравнивая эти уравнения с уравнениями (24) и (36) при $t=0$, выражаем $\check{\mathcal{K}}_{ \pm}$и $\check{\mathcal{R}}$ через $\mathcal{K}_{ \pm}$и $\mathcal{R}$, получая вторую и третью пару равенств в $(37)$. Временна́я эволюция для $K_{ \pm}$получается при подстановке выражения для $\chi_{ \pm}$из $(37)$ в формулу (34).

Аналогичным образом можно получить явную временну́ю зависимость спектральных данных в том случае, когда потенциал $v$ эволюционирует в соответствии с другими уравнениями иерархии (7).

\section{4. ОДНОПАРАМЕТРИЧЕСКИЕ СЕМЕЙСТВА КОММУТИРУЮЩИХ ДИНАМИЧЕСКИХ СИСТЕМ}

Хорошо известно (см., например, книгу [19]), что линейные ДУЧП первого порядка типа (3), (11), (15) тесно связаны с системами обыкновенных дифференциальных 
уравнений, описывающими их характеристические кривые. Например, динамические системы, ассоциированные с однопараметрическим семейством векторных полей $\hat{L}, \widehat{M}(3)$, суть

$$
\begin{array}{ll}
\hat{L}: & \frac{d x}{d y}=p+v_{x}(x, y, t), \\
\widehat{M}: & \frac{d x}{d t}=p^{2}+p v_{x}(x, y, t)-v_{y}(x, y, t) .
\end{array}
$$

Поэтому уравнение (1) характеризует класс функиий $v$, для которых две динамические системы первого порядка (41) коммутируют для любого р.

Существует также глубокая взаимосвязь между рассмотренным выше обратным спектральным преобразованием и теорией рассеяния, зависящей от времени $(y)$, для коммутирующих потоков (41). Пусть $\phi(x, y, t, p)$ - общая собственная функция операторов $\hat{L}$ и $\widehat{M}$, определенная формулой (38); тогда, решая систему $\omega=\phi(x, y, t, p)$ относительно $x$ (предполагая локальную обратимость), получаем следующее общее решение уравнений (41):

$$
\omega=\varphi(x, y, t ; p)-p^{2} t \quad \Leftrightarrow \quad x=r(\omega, y, t ; p) \sim p y+p^{2} t+\omega, \quad y \sim-\infty .
$$

Предел решения $r(\omega, y, t ; p)$ при $y=+\infty$

$$
x \sim p y+p^{2} t+\Omega(\omega, p), \quad y \sim+\infty,
$$

определяет зависящие от времени $(y)$ данные рассеяния $\Delta(\omega, p)=\Omega(\omega, p)-\omega$ первой динамической системы в (41), которые могут быть связаны с данными обратного спектрального преобразования $\mathcal{S}$ посредством обращения системы $\omega=\mathcal{S}(x-p y-$ $\left.p^{2} t, p, 0\right)$ относительно $x$ :

$$
\omega=\mathcal{S}\left(x-p y-p^{2} t, p, 0\right) \quad \Leftrightarrow \quad x-p y-p^{2} t=\Omega(\omega, p) .
$$

Побочным продуктом развитого в этой работе метода ОЗР является возможность восстановления функции $v$ по данным рассеяния $\Delta(\omega, p)$ для однопараметрического семейства динамических систем, заданных первым из уравнений (41).

Аналогичные рассмотрения могут быть проведены для динамических систем, ассоциированных со всей иерархией однопараметрического семейства операторов $\hat{L}, \widehat{M}_{n}, n \in \mathbb{N}$.

Благодарности. Данное исследование было поддержано грантом INFN 2006 г. и РФФИ (гранты № 04-01-00508, 06-01-90840 и 06-01-92053).

\section{Список литературы}

[1] S. V. Manakov, P. M. Santini, Phys. Lett. A, 359 (2006), 613-619; nlin.SI/0604024.

[2] С. В. Манаков, П.В. Сантини, Писъма в ЖЖЭТФ, 83:10 (2006), 534-538; nlin.SI/0604023.

[3] M. V. Pavlov, J. Math. Phys., 44 (2003), 4134-4156.

[4] E. V. Ferapontov, K. R. Khusnutdinova, Commun. Math. Phys., 248 (2004), 187-206. 
[5] M. Dunajski, J. Geom. Phys., 51 (2004), 126-137.

[6] V.E. Zakharov, Dispersionless limit of integrable systems in $2+1$ dimensions, Singular Limits of Dispersive Waves (Lyon, France, 1991), NATO Adv. Sci. Inst. Ser. B Phys., 320, ed. N. M. Ercolani et al., Plenum, New York, 1994.

[7] I. M. Krichever, Commun. Pure Appl. Math., 47 (1994), 437-475.

[8] K. Takasaki, T. Takebe, Rev. Math. Phys., 7 (1995), 743-808.

[9] M. Dunajski, L. J. Mason, P. Tod, J. Geom. Phys., 37 (2001), 63-93.

[10] B. Konopelchenko, L. Martínez Alonso, O. Ragnisco, J. Phys. A, 34 (2001), 10209-10217.

[11] F. Guil, M. Mañas, L. Martínez Alonso, J. Phys. A, 36 (2003), 6457-6472; nlin.SI/0211020.

[12] J. F. Plebañski, J. Math. Phys., 16 (1975), 2395-2402.

[13] B. Fuchssteiner, A. S. Fokas, Phys. D, 4 (1981), 47-66.

[14] F. Magri, J. Math. Phys., 19 (1978), 1156-1162.

[15] P. M. Santini, P. M. Fokas, Commun. Math. Phys., 115 (1988), 375-419.

[16] S. R. Deans, The Radon Transform and Some of Its Applications, 2nd ed., Krieger Publ., Malabar, FL, 1993.

[17] S. V. Manakov, V.E. Zakharov, Lett. Math. Phys., 5 (1981), 247-253.

[18] S. V. Manakov, Phys. D, 3 (1981), 420-427.

[19] R. Courant, D. Hilbert, Methods of Mathematical Physics. Vol. II. Partial Differential Equations, Intersci. Publ., Wiley, New York-London, 1962. 